\title{
Development and validation of a novel prognostic nomogram including tumor deposits could better predict survival for colorectal cancer: a population-based study
}

\author{
Rui Bai ${ }^{1,2 \#}$, Yinuo Tan ${ }^{1 \#}$, Dan $\mathrm{Li}^{1 \#}$, Mengyuan Yang ${ }^{1}$, Linzhen Yu ${ }^{1}$, Ying Yuan ${ }^{1,2}$, Xuefeng Fang ${ }^{1}$ \\ ${ }^{1}$ Department of Medical Oncology, Key Laboratory of Cancer Prevention and Intervention, Ministry of Education, the Second Affiliated Hospital of \\ Zhejiang University School of Medicine, Hangzhou, China; ${ }^{2}$ Cancer Institute, Key Laboratory of Cancer Prevention and Intervention, Ministry of \\ Education, the Second Affiliated Hospital of Zhejiang University School of Medicine, Hangzhou, China \\ Contributions: (I) Conception and design: All authors; (II) Administrative support: Y Yuan, X Fang; (III) Provision of study materials or patients: R \\ Bai, Y Tan, D Li; (IV) Collection and assembly of data: M Yang, L Yu; (V) Data analysis and interpretation: R Bai, Y Tan, D Li; (VI) Manuscript \\ writing: All authors; (VII) Final approval of manuscript: All authors. \\ \#These authors contributed equally to this work. \\ Correspondence to: Xuefeng Fang. Department of Medical Oncology, The Second Affiliated Hospital of Zhejiang University School of Medicine, \\ Hangzhou 310009, China. Email: xffang@zju.edu.cn.
}

Background: The number of tumor deposits (TDs) in colorectal cancer (CRC) prognosis remains debated. We evaluated whether the number of TDs affects prognosis in stage III CRC patients.

Methods: Univariate and multivariate analyses were performed with Cox proportional hazards models. The Kaplan-Meier method was used to estimate survival curves. The best cutoff was determined using $\mathrm{X}$-Tile. Patients were 1:1 randomly divided into the training set or the testing set. Prognostic nomogram was established for stage III CRC patients. Concordance index (C-index) and calibration plot were used to assess Nomogram models.

Results: In total, 18,043 (84.69\%) CRC patients without TDs and 3,263 (15.31\%) patients with TDs were analyzed. Patients with TDs had significantly worse cancer-specific survival (CSS) rates $(\mathrm{P}<0.001)$. The number of TDs is an independent factor for the CSS of stage III CRC patients. CSS nomogram of stage III CRC patients was constructed based on race, age at diagnosis, tumor location, histological grade, pathological type, T, N, TDs, chemotherapy. In training set, C-index for CSS nomogram 0.762 (95\% CI: 0.752-0.772). In testing set, the C-index for CSS nomogram 0.759 (95\% CI: 0.749-0.768). The quality of calibration plots of nomogram models was high.

Conclusions: The presence of TDs is an independent risk prognostic factor for stage III CRC. The number of TDs had a high proportion of prognostic impact.

Keywords: Colorectal cancer (CRC); tumor deposits (TDs); prognosis; stage III; nomogram

Submitted Jun 16, 2020. Accepted for publication Jan 25, 2021.

doi: 10.21037/atm-20-4728

View this article at: http://dx.doi.org/10.21037/atm-20-4728

\section{Introduction}

Colorectal cancer (CRC) is the third most common malignant tumor in the United States and the fifth most common in China $(1,2)$. The stage of CRC has a great impact on its treatment. CRC is treated with different postoperative strategies depending on the stage. Generally, stage III and high-risk stage II CRC patients receive postoperative adjuvant chemotherapy.

Tumor deposits (TDs) are discrete cancerous nodules located around the pericolic or perirectal fat or adjacent 
mesentery, with no obvious features of lymph node tissue or vascular structure. The differences between TDs and lymph node tissue are no recognizable lymph node structure in TDs. The latest version of the American Joint Committee on Cancer (AJCC) 8th edition of the TNM staging system has acknowledged the potential of the influence of TDs on CRC patients' survival outcome. As a reference factor for a high recurrence rate and poor prognosis, TDs increase the clinical doctor's awareness of the influence of adjuvant therapy selection. The AJCC 8th edition staging system states that TDs are a poor prognostic factor and continues to classify TDs as N1c while making it clear that TDs do not change the $\mathrm{T}$ stage of primary tumors. If there is no regional lymph node metastasis, TDs will change the $\mathrm{N}$ stage $(\mathrm{N} 0 \rightarrow \mathrm{N} 1 \mathrm{c})$, and if there is regional lymph node metastasis, the number of TDs does not need to be calculated with the number of positive lymph nodes.

TDs are an important factor in CRC prognosis (3-5). The description of TDs in the AJCC 5 th edition staging system is as follows: size, $>3.0 \mathrm{~mm}$; TDs are classified as lymph node metastasis, and primary TDs (PTDs) $\leq 3.0 \mathrm{~mm}$ are classified as continuous stage Dukes B adenocarcinoma (6). The description of TDs in the AJCC 6th edition staging system is contour; if there are nodules in the connective tissue of the lymphatic drainage area of the original tumor, there is no evidence of residual histology (7). TDs with regular and smooth shapes are classified as $\mathrm{pN}$ (regional lymph node metastases), while those with irregular shapes are classified as $\mathrm{pT}$ or $\mathrm{V} 1$ (microvein invasion) or V2 (visible to the unaided eye) (considered venous invasion) (8). In the AJCC 7th edition staging system, TDs are classified as a new type of metastatic nodule, pN1c (9). These factors of morphological size are difficult to study with data records. The number of TDs is recorded in the Surveillance, Epidemiology, and End Results (SEER) database. Whether the number of TDs affects the prognosis of CRC patients needs further discussion. Previous studies performed nomogram analysis of CRC stage III combined with TD stage (10). However, there is no nomogram model to show the impact of the number of TDs on the overall score. Our study developing and validating prognostic nomogram of stage III CRC patients and stage III CRC patients with TDs showed the influence of TD number on prognosis.

In this study, we focused on the effect of TDs on the prognosis of CRC stage III patients and used X-Tile software to distinguish the cutoff for the number of TDs for prognosis. Nomogram showed that the number of TDs in stage III CRC patients had a high impaction. We obtained a better understanding of the impact of the number of TDs on CRC prognosis.

We present the following article in accordance with the TRIPOD reporting checklist (available at http://dx.doi. org/10.21037/atm-20-4728).

\section{Methods}

\section{Patients and data sources}

A total of 139,166 CRC patients were selected from the SEER database (http://seer.cancer.gov/). Stage was regrouped into the AJCC 8th edition staging system from the AJCC 7th edition staging system. The inclusion criterion was a diagnosis of stage III CRC between 2010 and 2015, using reference number 19987-Nov2018. The end of follow-up is November 2018. The site codes C18.0, C18.218.7, C19.9 and C20.9 were used. The histology codes were adenocarcinoma [8140-8147, 8210-8211, 8220-8221, and 8260-8263], mucinous adenocarcinoma [8480-8481] and signet ring cell carcinoma (8490). Left-sided CRCs included C18.5-splenic flexure of colon, C18.6-descending, C18.7sigmoid colon, C19.9-rectosigmoid junction, and C20.9rectum. Right-sided CRCs included C18.0-cecum, C18.2ascending colon, C18.3-hepatic flexure of the colon, and C18.4-transverse colon. Chemotherapy information was also used for analysis.

The exclusion criteria were any of the following characteristics: missing or incomplete data on: (I) the number of TDs, (II) T, N, or M stage, (III) grade, (IV) tumor histology, (V) follow-up, (VI) no surgery, (VII) nonpathological confirmation of lymph node metastasis or neoadjuvant chemotherapy, and (VIII) more than one primary tumor. Complete-case analysis was used. Ultimately, 21,306 patients were identified. Patients 1:1 randomly divided into the training set or the testing set. The study was conducted in accordance with the Declaration of Helsinki (as revised in 2013).

\section{Statistical analysis}

The chi-square test was used to compare categorical variables of demographic and clinicopathological characteristics. The Kaplan-Meier method and log-rank test were used to compare differences in survival. Cancerspecific survival (CSS) was defined as the date of diagnosis to the date of death due to cancer. 
The cutoff point for the number of TDs was analyzed using X-Tile software, version 3.6.1 (http://www.tissuearray. org). X-Tile identified the cutoff with the minimum $\mathrm{P}$ values from log-rank $\chi^{2}$ statistics and categorized TDs in terms of survival.

Cox proportional hazards models were used to perform univariate and multivariate analyses. Hazard ratios (HRs) and $95 \%$ confidence intervals (CIs) were calculated. CSS nomogram of stage III CRC patients was constructed based on race, age at diagnosis, tumor location, histological grade, pathological type, T, N, TDs, chemotherapy. The statistical analyses and nomogram based on prognostic risk factors was established for predict 1-, 3- and 5-year CSS were performed with R statistical software, version 3.6.2 (http:// cran.r-project.org/).

Concordance index (C-index) were used to evaluate the predictive value of nomogram. Using a 45-degree line as an optimal model, calibration plots were used for the comparison between nomogram-predicted CSS and actual CSS.

\section{Results}

\section{Baseline characteristics of stage III CRC patients}

In total, 21,306 patients were included in this research, of which 10,653 were randomly assigned to the training set or the testing set (Figure 1).

The baseline characteristics of CRC patients with stage III according to the TDs status are shown in Table 1; 3,263 of whom had TDs (15.31\%). There was no significant difference in sex, race, age at diagnosis, histological grade, pathological type or $\mathrm{N}$ stage between the CRC with TDs group and the CRC without TDs group. Regarding the AJCC 8th edition T stage, the CRC with TDs group was associated with a higher $\mathrm{T}$ stage than the CRC without TDs group $(\mathrm{P}<0.001)$. The proportion of $\mathrm{CRC}$ patients with TDs in stages T3 and T4 was higher than that in CRC patients without TDs (T3 66.01\% vs. 64.86\%, T4 24.46\% vs. $16.54 \%)$. The proportion of CRC patients with TDs in stages IIIB and IIIC was higher than that in CRC patients without TDs (IIIB $69.81 \%$ vs. $65.96 \%$, IIIC $22.00 \%$ vs. $17.56 \%)$. The proportion of CRC patients with TDs on left side was higher than that in CRC patients without TDs $(\mathrm{P}<0.030,7.42 \%$ vs. $6.38 \%)$. A lower percentage of CRC patients with TDs received chemotherapy $(\mathrm{P}<0.001$, $60.77 \%$ vs. $64.05 \%)$.

\section{Prognostic factors of TDs from different lymph nodes}

The prognostic factors of TDs for CSS were investigated by Kaplan-Meier analysis. CRC patients with TDs had significantly poor CSS rates (Figure $2 A, \mathrm{P}<0.001$ ). The prognosis of CRC patients with TDs was significantly worse than that of CRC patients without TDs in both the $\mathrm{N} 1$ and $\mathrm{N} 2$ groups (Figure $2 B, \mathrm{P}<0.001$ ). The prognosis of the N2 with TDs group was worse than that of the nonN1c N1 with TDs group, and the prognosis of the nonN1c N1 with TDs group was worse than that of the N1c group (Figure $2 C, \mathrm{P}<0.001$ ).

To determine the significance of the number of TDs on CSS, we used X-Tile software to determine the best cutoff for the number of TDs. We determined that $1 \mathrm{TD}$ was the best cutoff for dichotomy and " 1 " " 5 "was the best cutoff for trichotomy. Then, we used Kaplan-Meier analysis, and the prognosis of the $>1 \mathrm{TD}$ group was worse than that of the $=1$ TD group (Figure 2D, $\mathrm{P}<0.001$ ). The prognosis of the TDs $\geq 5$ group was the worst, followed by the TDs $2-4$ group, and the $\mathrm{TD}=1$ group was the best (Figure $2 E, \mathrm{P}<0.001$ ).

\section{Clinical and tumor characteristics between training set and testing set}

There was no significant difference in sex, race, age at diagnosis, tumor location, histological grade, pathological type, T, N stage, chemotherapy or TDs between the training set and testing set (Table 2).

\section{Identification of independent prognostic factors for CSS in stage III CRC patients (training set)}

Univariate and multivariate analyses were conducted to test the prognostic differences in CSS between stage III CRC patients in the training set. In the multivariate analysis, in addition to the number of TDs $(0 / 2-4$ $\mathrm{P}<0.001 / \geq 5 \mathrm{P}<0.001)$, other significant prognostic factors, including race (white/black $\mathrm{P}<0.001$ /other $\mathrm{P}=0.028$ ), age at diagnosis $(<65 / \geq 65$ years, $\mathrm{P}<0.001)$, tumor location (left/ right, $\mathrm{P}=0.003$ ), histological grade (well differentiated/ moderately differentiated $\mathrm{P}=0.498$ /poorly differentiated $\mathrm{P}=0.003$ /undifferentiated, $\mathrm{P}<0.001)$, pathological type (adenocarcinoma/signet ring cell carcinoma, $\mathrm{P}=0.001$ ), T stage (T1/T2 $\mathrm{P}=0.030 / \mathrm{T} 3 \mathrm{P}<0.001 / \mathrm{T} 4 \mathrm{P}<0.001), \mathrm{N}$ stage $(\mathrm{N} 1 / \mathrm{N} 2 \mathrm{P}<0.001)$ and chemotherapy $(\mathrm{P}<0.001)$ were identified. All significant prognostic factors identified by the 


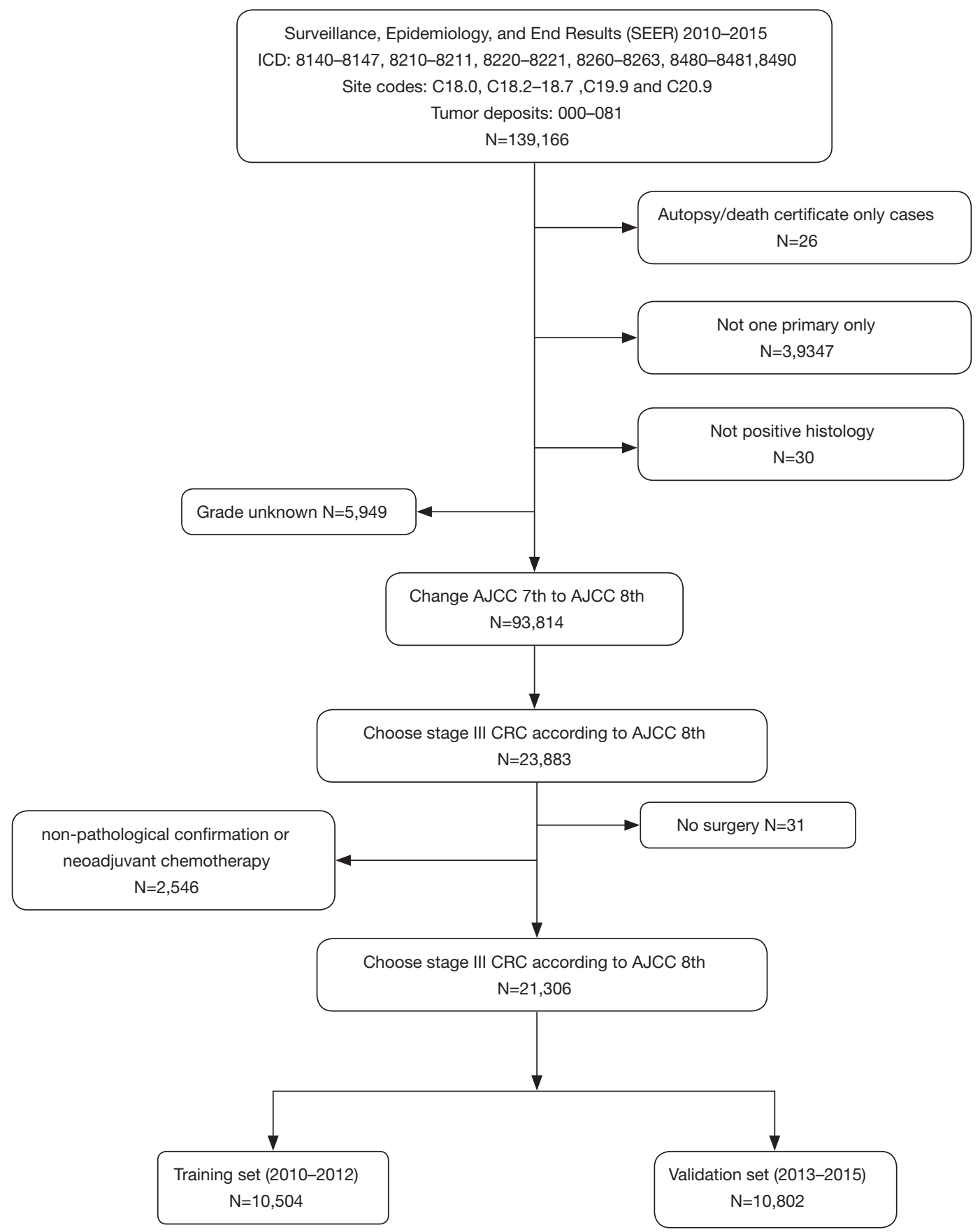

Figure 1 Flow chart of patient selection. CRC, colorectal cancer. 
Table 1 Clinical and tumor characteristics between patients with TDs and patients without TDs

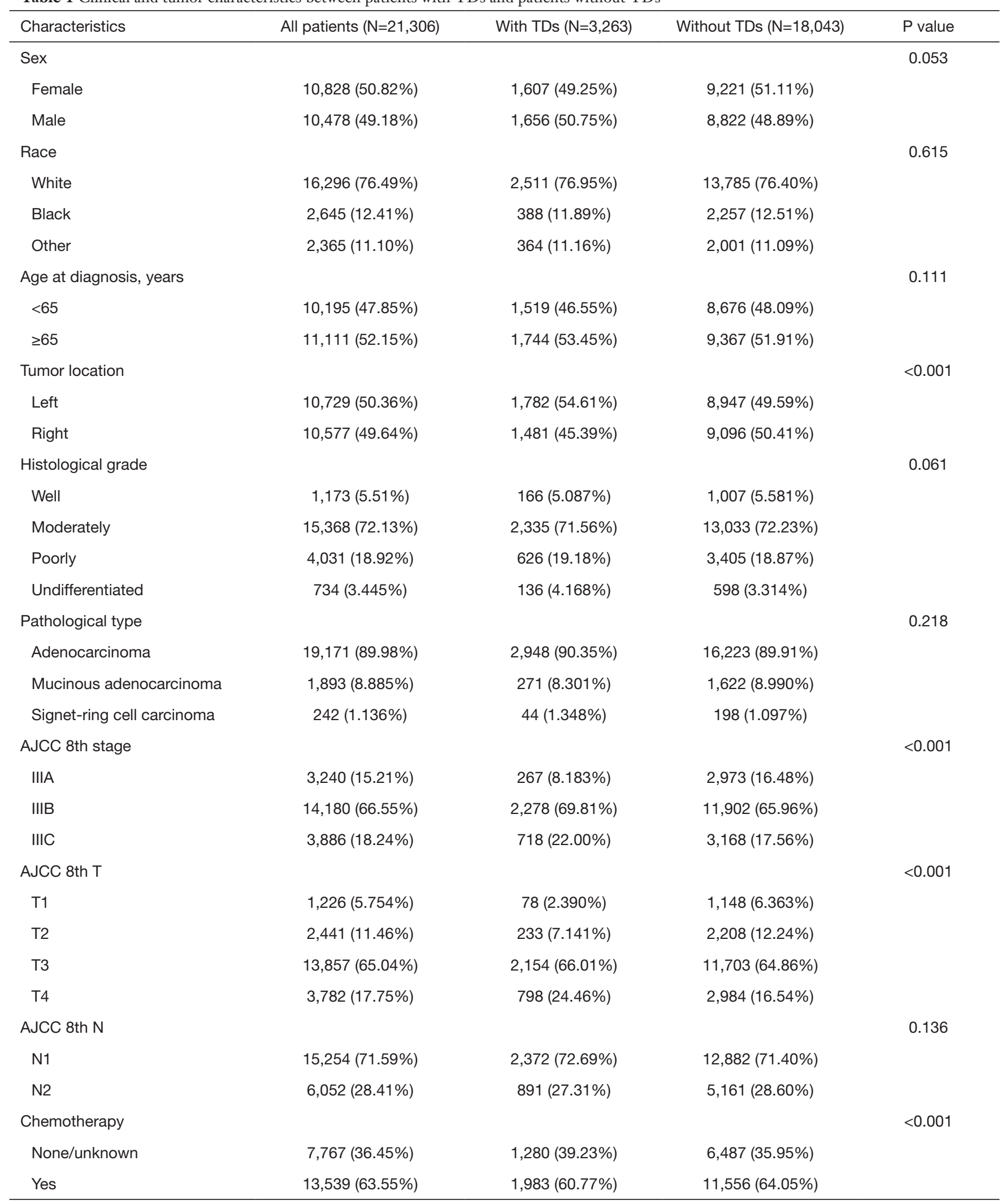

TD, tumor deposits. 


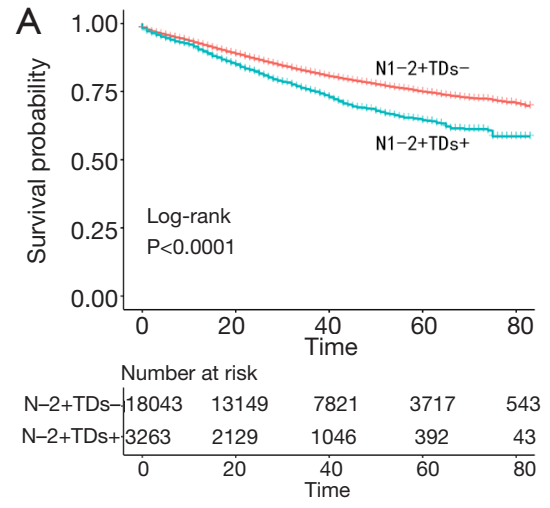

D

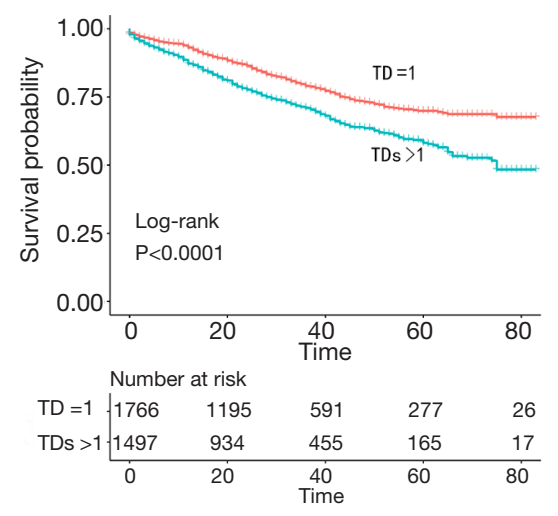

B

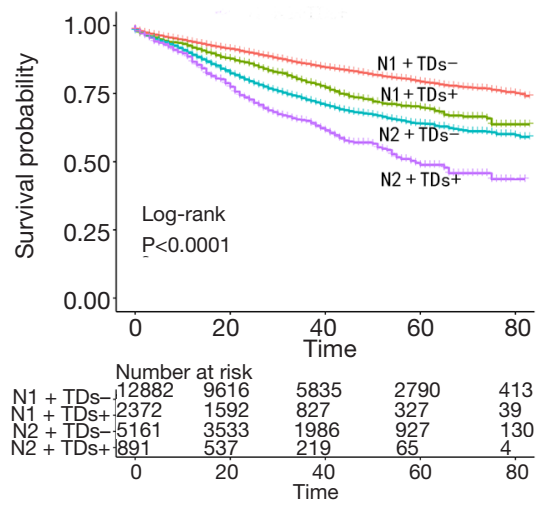

E

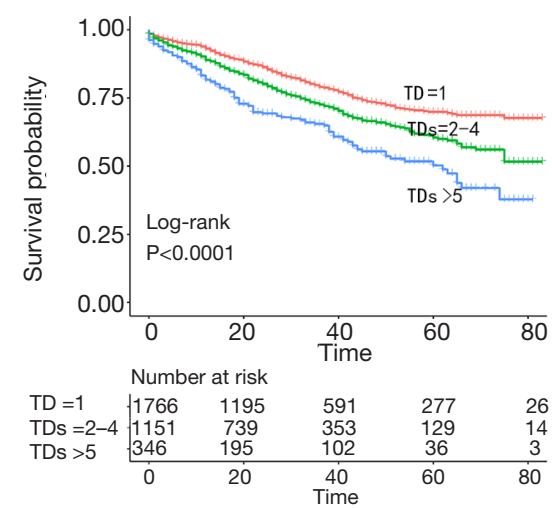

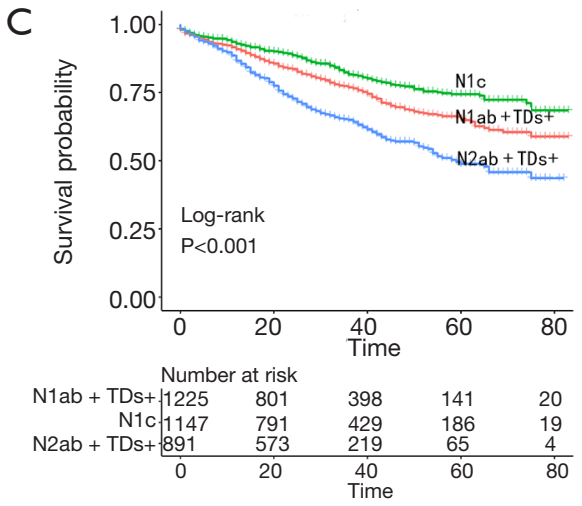

Figure 2 The Kaplan-Meier method was used to estimate survival curves. (A) Kaplan-Meier analysis of CRC patients with and without TDs. (B) Kaplan-Meier analysis of stage N1 and N2 CRC patients with and without TDs. (C) Kaplan-Meier analysis of stage N2 patients with TDs, non-N1c N1 patients with TDs and N1c patients. (D) Kaplan-Meier analysis of the $>1$ and =1 TD groups. (E) Kaplan-Meier analysis of the $\geq 5,2-4$ and $=1$ TD groups. CRC, colorectal cancer; TD, tumor deposits.

univariate analysis were included in the multivariate Cox regression analysis. Sex was not revealed as an independent prognostic factor. (Table 3).

\section{Construction of nomogram and nomogram validation}

CSS nomogram of stage III CRC patients was constructed based on race, age at diagnosis, tumor location, histological grade, pathological type, T, N, TDs, chemotherapy (Figure 3).

The CSS nomograms of stage III CRC patients were validated. In training set, the C-index for the CSS nomogram 0.762 (95\% CI: $0.752-0.772$ ). In the testing set, the C-index for the CSS nomogram 0.759 (95\% CI: 0.7490.768). High quality of calibration plots in CSS nomogram models had been identified (Figure 4).

\section{Clinical and tumor characteristics of patients with TDs}

Among the 3,263 patients with TDs, 1,766 had =1 TD, 1151 had 2-4 TDs, and 346 had $\geq 5$ TDs. There was no significant difference in sex, age at diagnosis, pathological type, radiation or chemotherapy between the CRC with 2-4 TDs group and the CRC with $=1$ TD group. It seems that there were more whites in the 2-4 TDs group than in the $=1$ TD group (78.63\% vs. $75.48 \%)$. Regarding the AJCC 8th edition staging system, CRC patients with 2-4 TDs were associated with a higher $\mathrm{T}$ and $\mathrm{N}$ stage than CRC patients with $1 \mathrm{TD}(\mathrm{P}<0.001)$. Compared to the CRC with $=1$ TD group, the CRC with 2-4 TDs group was associated with more TDs located on the left side $(\mathrm{P}<0.001$, $57.78 \%$ vs. 52.04\%). The proportion of CRC patients with 2-4 TDs and poorly and undifferentiated diseases was higher than that in CRC patients with $=1 \mathrm{TD}(\mathrm{P}<0.001$, 
Table 2 Clinical and tumor characteristics between training set and testing set

\begin{tabular}{|c|c|c|c|}
\hline Characteristics & Training set & Testing set & $P$ value \\
\hline Female & $5,434(51.01 \%)$ & $5,394(50.63 \%)$ & \\
\hline Male & $5,219(48.99 \%)$ & $5,259(49.37 \%)$ & \\
\hline Race & & & 0.884 \\
\hline Other & $1,187(11.14 \%)$ & $1,178(11.06 \%)$ & \\
\hline Age at diagnosis, years & & & 0.1 \\
\hline$<65$ & $5,158(48.42 \%)$ & $5,037(47.28 \%)$ & \\
\hline$\geq 65$ & $5,495(51.58 \%)$ & $5,616(52.72 \%)$ & \\
\hline Right & $5,286(49.62 \%)$ & $5,291(49.67 \%)$ & \\
\hline Histological grade & & & 0.675 \\
\hline Well & $586(5.501 \%)$ & $587(5.510 \%)$ & \\
\hline Moderately & 7,680 (72.09\%) & 7,688 (72.17\%) & \\
\hline Poorly & $2,004(18.81 \%)$ & 2,027 (19.03\%) & \\
\hline Undifferentiated & $383(3.595 \%)$ & $351(3.295 \%)$ & \\
\hline Pathological type & & & 0.415 \\
\hline Adenocarcinoma & $9,612(90.23 \%)$ & $9,559(89.73 \%)$ & \\
\hline T3 & 6,934 (65.09\%) & 6,923 (64.99\%) & \\
\hline $\mathrm{T} 4$ & $1,852(17.38 \%)$ & $1,930(18.12 \%)$ & \\
\hline $\mathrm{N}$ & & & 0.332 \\
\hline N1 & 7,625 (71.58\%) & 7,629 (71.61\%) & \\
\hline N2 & $3,028(28.42 \%)$ & 3,024 (28.39\%) & \\
\hline Chemotherapy & & & 0.887 \\
\hline No/unknown & $3,889(36.51 \%)$ & $3,878(36.40 \%)$ & \\
\hline Yes & $6,764(63.49 \%)$ & $6,775(63.60 \%)$ & \\
\hline TDs & & & 0.299 \\
\hline 0 & $9,046(84.92 \%)$ & $8,997(84.46 \%)$ & \\
\hline 1 & $853(8.007 \%)$ & $913(8.570 \%)$ & \\
\hline $2-4$ & $1,151(5.402 \%)$ & $570(5.351 \%)$ & \\
\hline$\geq 5$ & $184(1.727 \%)$ & $162(1.521 \%)$ & \\
\hline
\end{tabular}

TD, tumor deposits. 
Table 3 Univariate and multivariate analysis of the factors, including TD status, associated with the CSS for CRC stage III in training set

\begin{tabular}{|c|c|c|c|c|c|c|}
\hline Characteristics & \multicolumn{3}{|c|}{ Univariate } & \multicolumn{3}{|c|}{ Multivariate } \\
\hline \multicolumn{7}{|l|}{ Sex } \\
\hline Female & 1 & & & 1 & & \\
\hline Male & 0.90 & $0.83-0.98$ & 0.014 & 1.03 & $0.95-1.13$ & 0.475 \\
\hline \multicolumn{7}{|l|}{ Race } \\
\hline Black & 1.16 & $1.03-1.32$ & 0.017 & 1.37 & $1.21-1.55$ & $<0.001$ \\
\hline Other & 0.80 & $0.69-0.92$ & 0.003 & 0.85 & $0.73-0.98$ & 0.028 \\
\hline \multicolumn{7}{|l|}{ Age at diagnosis, years } \\
\hline$<65$ & 1 & & & 1 & & \\
\hline Left & 1 & & & 1 & & \\
\hline Right & 1.61 & $1.48-1.76$ & $<0.001$ & 1.15 & $1.05-1.26$ & 0.003 \\
\hline \multicolumn{7}{|l|}{ Histological grade } \\
\hline Well & 1 & & & 1 & & \\
\hline Moderate & 1.21 & $0.97-1.50$ & 0.092 & 1.08 & $0.87-1.34$ & 0.498 \\
\hline Poorly & 2.06 & $1.64-2.58$ & $<0.001$ & 1.42 & $1.13-1.79$ & 0.003 \\
\hline Undifferentiated & 2.83 & $2.15-3.72$ & $<0.001$ & 1.78 & $1.35-2.35$ & $<0.001$ \\
\hline \multicolumn{7}{|l|}{ Pathological type } \\
\hline Adenocarcinoma & 1 & & & 1 & & \\
\hline T3 & 3.62 & $2.60-5.04$ & $<0.001$ & 2.60 & $1.86-3.63$ & $<0.001$ \\
\hline $\mathrm{T} 4$ & 8.31 & $5.94-11.62$ & $<0.001$ & 5.37 & $3.83-7.54$ & $<0.001$ \\
\hline \multicolumn{7}{|l|}{ AJCC 8th $\mathrm{N}$} \\
\hline N1 & 1 & & & 1 & & \\
\hline $\mathrm{N} 2$ & 1.92 & $1.76-2.10$ & $<0.001$ & 1.82 & $1.66-1.99$ & $<0.001$ \\
\hline \multicolumn{7}{|l|}{ TDs } \\
\hline 0 & 1 & & & 1 & & \\
\hline 1 & 1.11 & $0.94-1.31$ & 0.207 & 1.1 & $0.94-1.30$ & 0.239 \\
\hline $2-4$ & 1.64 & $1.39-1.93$ & $<0.001$ & 1.50 & $1.27-1.77$ & $<0.001$ \\
\hline$\geq 5$ & 2.55 & $2.01-3.22$ & $<0.001$ & 1.75 & $1.43-2.16$ & $<0.001$ \\
\hline \multicolumn{7}{|l|}{ Chemotherapy } \\
\hline None/unknown & 1 & & & 1 & & \\
\hline Yes & 0.37 & $0.34-0.40$ & $<0.001$ & 0.42 & $0.39-0.46$ & $<0.001$ \\
\hline
\end{tabular}

TD, tumor deposits; CSS, cancer-specific survival. 


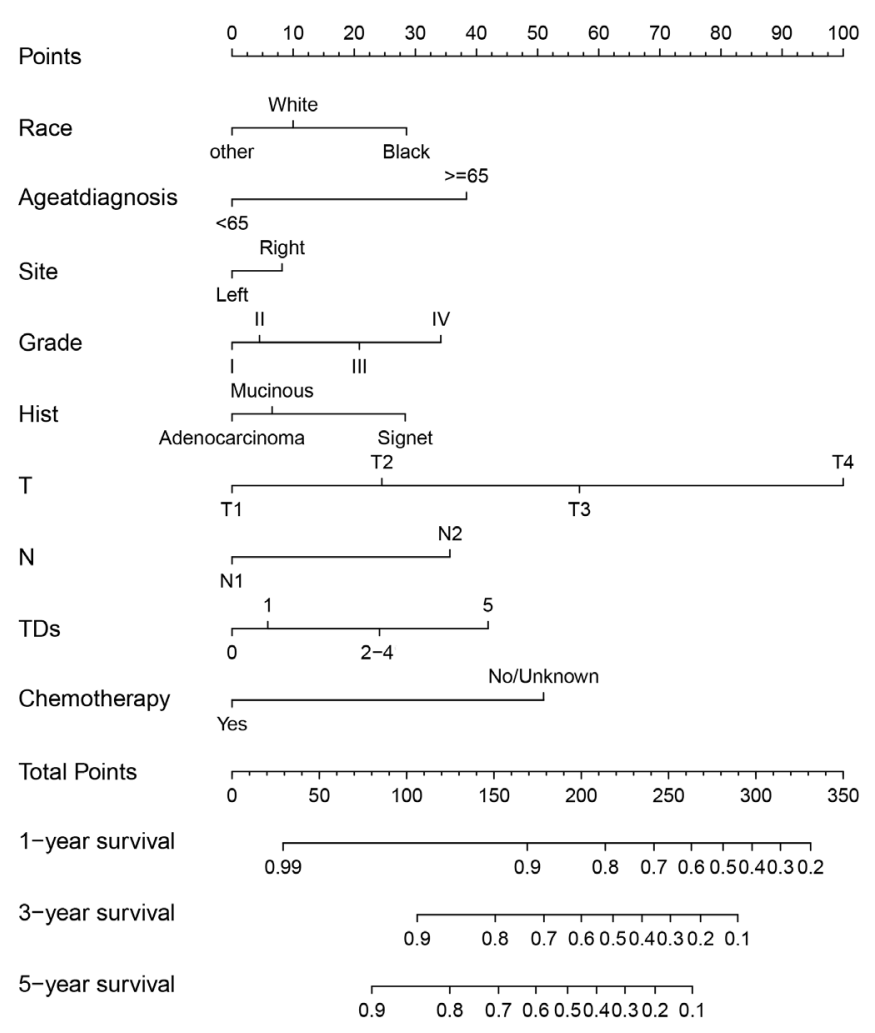

Figure 3 CSS nomograms in stage III CRC patients in 1-, 3- and 5-year CSS. CRC, colorectal cancer; CSS, cancer-specific survival.

$19.46 \%$ vs. $18.01 \%$ and $5.734 \%$ vs. $2.831 \%$ ) (Table 3).

There was no significant difference in sex, age at diagnosis, tumor location, radiation or chemotherapy between the CRC with $\geq 5$ TDs group and the CRC with $=1$ TD group. There were more whites in the $\geq 5$ TDs group than in the $=1$ TD group $(78.90 \%$ vs. $75.48 \%)$. CRC patients with $\geq 5$ TDs were associated with a higher $\mathrm{T}$ and $\mathrm{N}$ stage than CRC patients with $1 \mathrm{TD}(\mathrm{P}<0.001)$. Compared to the CRC with $=1$ TD group, the CRC with $\geq 5$ TDs group was associated with more mucinous or signet-ring cell carcinoma $(\mathrm{P}<0.013,12.43 \%$ vs. $7.871 \%$, $1.736 \%$ vs. $1.133 \%)$. The proportion of CRC patients with $\geq 5$ TDs and poorly and undifferentiated diseases was higher than that in CRC patients with $=1 \mathrm{TD}(\mathrm{P}<0.001,24.28 \%$ vs. $18.01 \%$ and $5.780 \%$ vs. $2.831 \%$ ) (Table 4 ).

\section{Univariate and multivariate analysis of the factors associated with CSS for stage III CRC patients with TDs}

Univariate and multivariate analyses were conducted to test the prognostic differences in CSS between stage III CRC patients with and without TDs, including the TD status $(=1 / 2-4 / \geq 5)$. In the univariate analysis, in addition to the TDs status $(\mathrm{P}<0.001)$, other significant prognostic factors, including sex (female/male, $\mathrm{P}=0.021$ ), age at diagnosis $(<65 / \geq 65$ years, $\mathrm{P}<0.001)$, tumor location (left/ right, $\mathrm{P}<0.001$ ), histological grade (well differentiated/ moderately differentiated $\mathrm{P}=0.430$ /poorly differentiated $\mathrm{P}=0.066 /$ undifferentiated, $\mathrm{P}=0.001$ ), pathological type (adenocarcinoma/mucinous adenocarcinoma $\mathrm{P}=0.031 /$ signet ring cell carcinoma, $\mathrm{P}=0.001)$, $\mathrm{T}$ stage ( $\mathrm{T} 1 / \mathrm{T} 2$ $\mathrm{P}=0.846 ; \mathrm{T} 3 \mathrm{P}=0.021 ; \mathrm{T} 4 \mathrm{P}<0.001)$, and $\mathrm{N}$ stage $(\mathrm{P}<0.001)$, were identified. All significant prognostic factors identified in the univariate analysis were included in the multivariate Cox regression analysis. Tumor location, grade, and pathological type were not identified as independent prognostic factors. The factors that remained independent prognostic factors were as follows: number of TDs (=1/2-4, HR: 1.29, 95\% CI: 1.10-1.51, $\mathrm{P}<0.001 / \geq 5$ HR: 1.74, 95\% CI: 1.41-2.14, $\mathrm{P}<0.001)$, histological grade (well differentiated/moderately differentiated $\mathrm{P}=0.442$ / poorly differentiated $\mathrm{P}=0.815 /$ undifferentiated, $\mathrm{P}=0.029)$, age at diagnosis $(<65 / \geq 65$ years, HR: $2.54,95 \%$ CI: $2.17-2.97$, $\mathrm{P}<0.001$ ), N stage (HR: 1.67, 95\% CI: 1.44-1.95, $\mathrm{P}<0.001$ ), $\mathrm{T}$ stage (T1/T2 HR:0.88, 95\% CI: 0.39-1.98 P=0.688/T3 HR: 


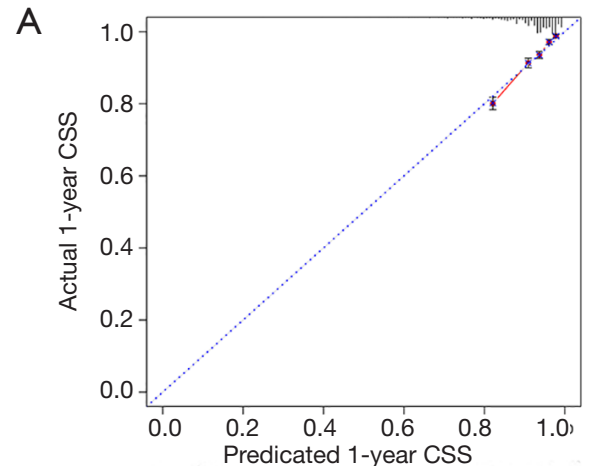

1 year, $n=10,653, d=2,121, P=16,2000$ subjects per group Gray: ideal

D

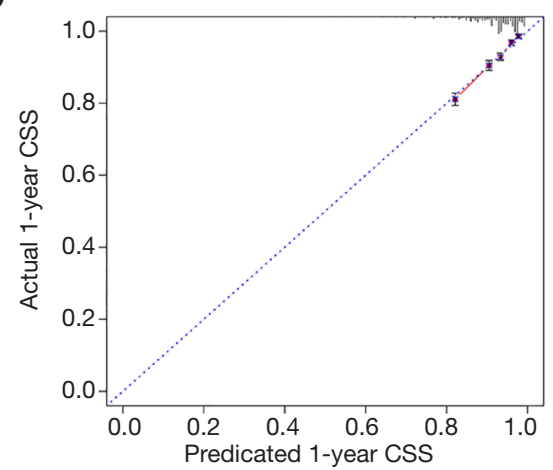

1 year, $n=10,653, d=2,091, P=14,2000$ subjects per group Gray: ideal $X$-resampling optimism added, $B=1,000$, based on observed-predict
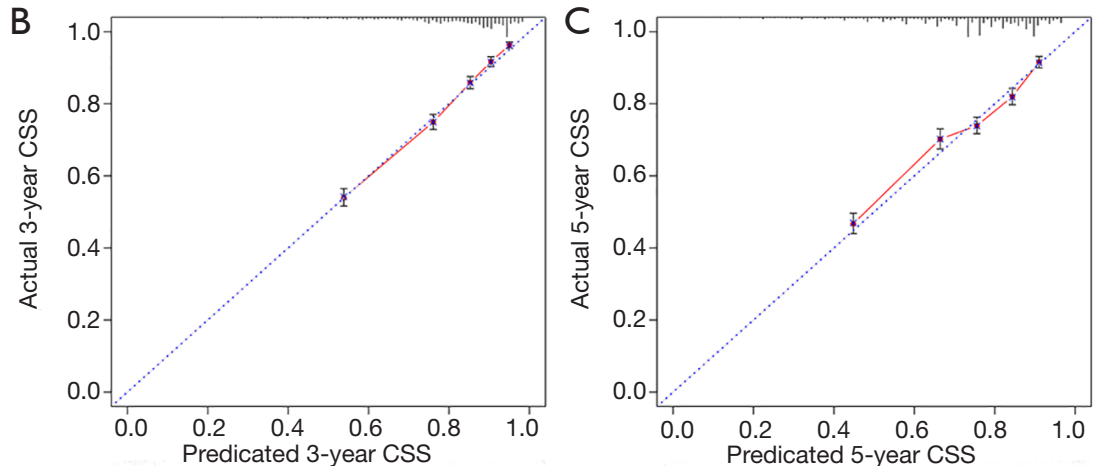

3 year, $n=10,653, d=2,121, P=18,2000$ subjects per group Gray: ideal 5 year, $n=10,653, d=2,121, P=16,2000$ subjects per group Gray: ideal $X$-resampling optimism added, $B=1,000$, based on observed-predicted $X$-resampling optimism added, $B=1,000$, based on observed-predicted

E

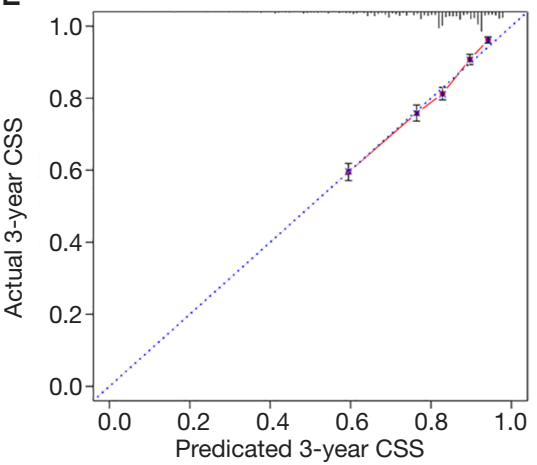

$F$

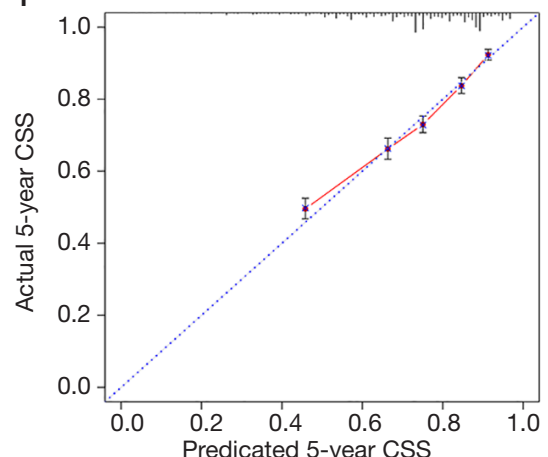

3 year, $n=10,653, d=2,091, P=14,2000$ subjects per group Gray: ideal $\quad 5$ year, $n=10,653, d=2,091, P=14,2000$ subjects per group Gray: ideal X-resampling optimism added, $B=1,000$, based on observed-predicted $\quad X$-resampling optimism added, $B=1,000$, based on observed-predicted

Figure 4 Calibration plots of CSS nomograms in training and testing sets. (A,B,C) Calibration plots of 1-, 3- and 5-year CSS in training set; (D,E,F) calibration plots of 1-, 3- and 5-year CSS in testing set. CSS, cancer-specific survival.

1.77, 95\% CI: 0.88-3.56 P=0.146/T4 HR: 3.56, 95\% CI: 1.76 $7.22 \mathrm{P}=0.001)$ and chemotherapy (HR: 0.44, 95\% CI: 0.38-0.51, $\mathrm{P}<0.001)$ (Table 5).

\section{Discussion}

In the current study, we used the SEER database to investigate the role of TDs and the number of TDs in stage III CRC. The large sample size and good representativeness of the United States patient population further improve the statistical reliability of our study and the universality of the results. In our population of stage III CRC patients, the incidence of TDs was $15.31 \%$. CRC patients with TDs had significantly poor CSS rates, consistent with previous studies $(11,12)$. The presence of both TDs and lymph nodes were associated with a worse prognosis than the presence of either TDs or lymph nodes alone. Additionally, our multivariable models confirmed the independent prognostic value of the number of TDs $(=1 / 2-4 / \geq 5)$ in stage III CRC patients.

Based on the anatomy of the TNM staging system, the concept of prognosis and predictors was introduced into the AJCC 7th edition CRC staging system (9). It was proposed that positive circumcision margins, lymphatic vessel infiltration, peripheral nerve infiltration and TDs could be assessed (13). The AJCC 8th edition staging system promotes the "prognosis and prediction" of CRC. On the basis of further refinement of the anatomy of TNM stage, an improved measurement system was introduced: the "prognosis and prediction" of nonanatomic factors based on histopathology and gene detection. The system also includes TDs; the serum CEA level and tumor regression score; circumcision margins; vascular lymphatic vessel infiltration; peripheral nerve infiltration; a microsatellite unstable state; the $K R A S, N R A S$ and $B R A F$ gene states; and the recommended level of evidence.

In the AJCC 8th edition staging system, the $\mathrm{T}$ stage was not updated; regarding the $\mathrm{N}$ stage, the definition 
Table 4 The clinical and tumor characteristics between patients with TDs ( $\mathrm{P}$ value compared to TD =1)

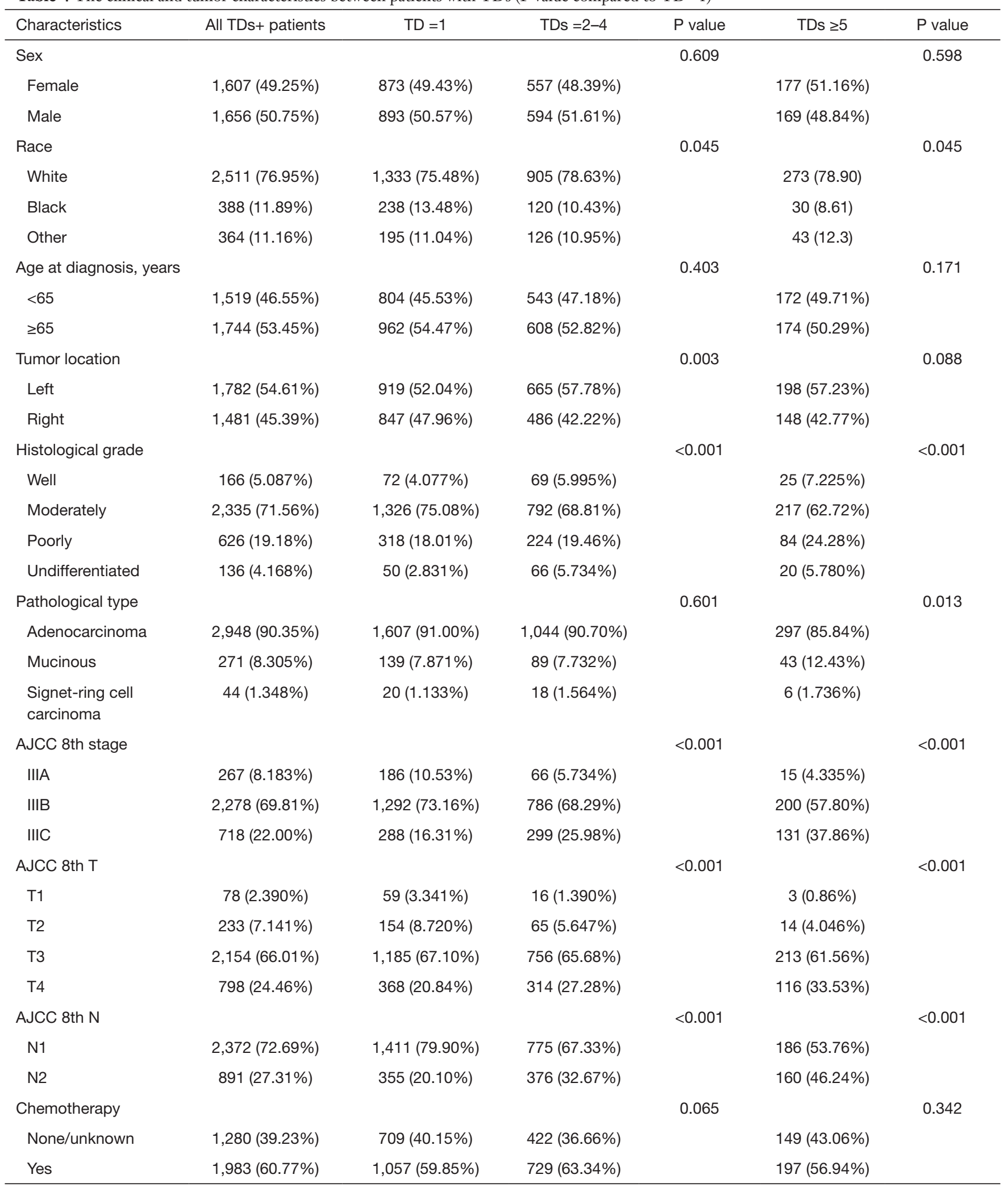

TD, tumor deposits. 
Table 5 Univariate and multivariate analysis of the factors, including TDs status (=1 or $=2-4$ or $\geq 5$ ) CSS for TDs+ CRC with stage III

\begin{tabular}{|c|c|c|c|c|c|c|}
\hline Characteristics & \multicolumn{3}{|c|}{ Univariate } & \multicolumn{3}{|c|}{ Multivariate } \\
\hline \multicolumn{7}{|l|}{ Sex } \\
\hline Female & 1 & & & 1 & & \\
\hline Male & 0.85 & $0.74-0.98$ & 0.021 & 0.93 & $0.81-1.07$ & 0.312 \\
\hline \multicolumn{7}{|l|}{ Race } \\
\hline Black & 1.13 & $0.91-1.39$ & 0.268 & & & \\
\hline Other & 0.94 & $0.75-1.18$ & 0.575 & & & \\
\hline \multicolumn{7}{|l|}{ Age at diagnosis, years } \\
\hline$<65$ & 1 & & & 1 & & \\
\hline Left & 1 & & & 1 & & \\
\hline Right & 1.44 & $1.25-1.66$ & $<0.001$ & 1.05 & $0.91-1.21$ & 0.531 \\
\hline \multicolumn{7}{|l|}{ Histological grade } \\
\hline Well & 1 & & & 1 & & \\
\hline Moderate & 0.88 & $0.64-1.21$ & 0.430 & 0.87 & $0.63-1.20$ & 0.410 \\
\hline Poorly & 1.37 & $0.98-1.92$ & 0.066 & 1.02 & $0.72-1.43$ & 0.921 \\
\hline Undifferentiated & 2.00 & $1.33-2.99$ & 0.001 & 1.58 & $1.05-2.39$ & 0.029 \\
\hline \multicolumn{7}{|l|}{ Pathological type } \\
\hline $\mathrm{T} 2$ & 0.92 & $0.41-2.06$ & 0.846 & 0.88 & $0.39-1.98$ & 0.763 \\
\hline T3 & 2.28 & $1.13-4.59$ & 0.021 & 1.77 & $0.88-3.56$ & 0.112 \\
\hline $\mathrm{T} 4$ & 4.90 & $2.43-9.90$ & $<0.001$ & 3.56 & $1.76-7.22$ & 0.001 \\
\hline \multicolumn{7}{|l|}{ AJCC 8th N } \\
\hline $\mathrm{N} 1$ & 1 & & & 1 & & \\
\hline $\mathrm{N} 2$ & 1.88 & $1.63-2.17$ & $<0.001$ & 1.89 & $1.62-2.20$ & $<0.001$ \\
\hline \multicolumn{7}{|l|}{ TDs } \\
\hline 1 & 1 & & & 1 & & \\
\hline $2-4$ & 1.44 & $1.23-1.67$ & $<0.001$ & 1.36 & $1.16-1.59$ & 0.001 \\
\hline$\geq 5$ & 2.15 & $1.76-2.63$ & $<0.001$ & 1.75 & $1.43-2.16$ & $<0.001$ \\
\hline \multicolumn{7}{|l|}{ Chemotherapy } \\
\hline None/unknown & 1 & & & 1 & & \\
\hline Yes & 0.42 & $0.37-0.49$ & $<0.001$ & 0.44 & $0.38-0.51$ & $<0.001$ \\
\hline
\end{tabular}

TD, tumor deposits; CRC, colorectal cancer; CSS, cancer-specific survival. 
of TDs was restated with level II evidence. TDs indicate the presence of lymphatic drainage in primary tumors or isolated tumor nodes in the region (within the adipose tissue of the mesocolon and mesorectum). Most TDs originate from vascular lymphatic invasion.

The value of the number of TDs in the prognosis of CRC tumors has been controversial (14). Some scholars believe that the prognosis cannot be distinguished by the number of TDs (15), but some scholars believe that the number of TDs may be an important factor for prognosis (8) and that it should be added to the $\mathrm{N}$ classification system (16). The number of TDs may be restaged after being added to the number of lymph nodes. Our results suggest that the prognosis of the TDs $\geq 5$ group was the worst, followed by the TDs 2-4 group, and the TD $=1$ group was the best. Why are the numbers so small? One possible explanation is that our current $\mathrm{N}$ stage $\mathrm{N} 1$ a represents 1 positive regional lymph node, N1b represents 2-3, N2a represents 4-6, and $\mathrm{N} 2 \mathrm{~b}$ represents more than 7 . It is well known that a onenode change in the total number of lymph nodes can alter the stage of CRC and have prognostic implications (17). The addition of more TDs to the lymph node stage did not have a greater effect on the $\mathrm{N}$ stage. Increasing the number of TDs to the lymph nodes may change the specific stage of the lymph nodes.

Song (18) classified cancer nodules as a new stage (nPN) after lymph node metastasis was included in the statistics, and nPN was superior to the AJCC 7th edition staging system stage in assessing patient prognosis. Ueno et al. (19) examined extraintestinal cancer nodules in a total of 3,958 patients and showed that the classification of cancerous nodules (irrespective of shape) into $\mathrm{N}$ stages could simplify tumor staging criteria, enhance the objectivity of the prognostic assessment prognosis, and increase the accuracy of the prognostic assessment. Ueno et al. (20) demonstrated that a high number of $\geq 5$ TDs was correlated with poor survival. The same study also suggested that TDs and node-positive disease showed similar survival rates. Therefore, the AJCC 8th edition staging system recommended evaluating the number of TDs, specifically 1-4 TDs or $\geq 5$ TDs. In our study, we focused on stage III CRC patients with TDs, and 1 was the best cutoff for the number of TDs, which may be because we excluded factors such as neoadjuvant chemotherapy and targeted only patients with the pathological staging of the lymph nodes. Using X-Tile, we also found that, $\geq 5$ TDs could be used to distinguish the prognosis of patients with TDs. Multiple metastatic pathways in tumors will result in wide tumor spread. TDs numbers had been identified as significant independent factors in univariate/multivariate. This is the first nomogram model to consider the prognostic implication of the number of tumor deposits. We can use this nomogram model to predict prognosis risk after surgery. Patients with high risk can receive more aggressive treatment.

The current research has some limitations. The research is only based on the data of SEER from 2010 to 2015. The ethnic type is mainly white, and it needs to be verified by a large sample of multi-centers.

\section{Conclusions}

The number of TDs has predictive value for the prognosis of stage III CRC patients. The presence of TDs and the number of TDs should be considered when making treatment decisions.

\section{Acknowledgments}

The authors would like to thank the Surveillance, Epidemiology, and End Results (SEER) Program tumor registries in the creation of the SEER database.

Funding: This work was supported by grants from the National Natural Science Foundation of China (No. 81872481), Zhejiang Basic Public Welfare Research Project (LGF18H160040 to RB) and the Key Disciplines of Chinese Medicine (Integrated Traditional Chinese and Western Medicine) in Zhejiang Province during the Thirteenth Five-year Plan (2017-XK-A40).

\section{Footnote}

Reporting Checklist: The authors have completed the TRIPOD reporting checklist. Available at http://dx.doi. org/10.21037/atm-20-4728

Conflicts of Interest: All authors have completed the ICMJE uniform disclosure form (available at http://dx.doi. org/10.21037/atm-20-4728). The authors have no conflicts of interest to declare.

Ethical Statement: The authors are accountable for all aspects of the work in ensuring that questions related to the accuracy or integrity of any part of the work are appropriately investigated and resolved. The study was conducted in accordance with the Declaration of Helsinki 
(as revised in 2013). This study used previously collected deidentified data. The need for informed consent had been waived due to the retrospective nature of the study, and the study was deemed exempt from review by the Ethics Committee of the Second Affiliated Hospital of Zhejiang University School of Medicine.

Open Access Statement: This is an Open Access article distributed in accordance with the Creative Commons Attribution-NonCommercial-NoDerivs 4.0 International License (CC BY-NC-ND 4.0), which permits the noncommercial replication and distribution of the article with the strict proviso that no changes or edits are made and the original work is properly cited (including links to both the formal publication through the relevant DOI and the license). See: https://creativecommons.org/licenses/by-nc-nd/4.0/.

\section{References}

1. Siegel RL, Miller KD, Jemal A. Cancer statistics, 2019. CA Cancer J Clin 2019;69:7-34.

2. Chen $W$, Zheng $\mathrm{R}$, Baade $\mathrm{PD}$, et al. Cancer statistics in China, 2015. CA Cancer J Clin 2016;66:115-32.

3. Kawai K, Nozawa H, Hata K, et al. Nomogram Predicting Survival After Recurrence in Patients With Stage I to III Colon Cancer: A Nationwide Multicenter Study. Dis Colon Rectum 2018;61:1053-62.

4. Al Sahaf O, Myers E, Jawad M, et al. The prognostic significance of extramural deposits and extracapsular lymph node invasion in colon cancer. Dis Colon Rectum 2011;54:982-8.

5. Belt EJ, van Stijn MF, Bril H, et al. Lymph node negative colorectal cancers with isolated tumor deposits should be classified and treated as stage III. Ann Surg Oncol 2010;17:3203-11.

6. Puppa G, Colombari R, Pelosi G, et al. Pericolonic tumour deposits in colorectal cancer patients: the challenge is ongoing. Histopathology 2008;52:767-8; author reply 8-9.

7. Sobin LH. TNM, sixth edition: new developments in general concepts and rules. Semin Surg Oncol 2003;21:19-22.

8. Nagtegaal ID, Knijn N, Hugen N, et al. Tumor Deposits in Colorectal Cancer: Improving the Value of Modern Staging-A Systematic Review and Meta-Analysis. J Clin Oncol 2017;35:1119-27.

9. Tong LL, Gao P, Wang ZN, et al. Is the seventh edition of the UICC/AJCC TNM staging system reasonable for patients with tumor deposits in colorectal cancer? Ann
Surg 2012;255:208-13.

10. Zheng P, Chen Q, Li J, et al. Prognostic Significance of Tumor Deposits in Patients With Stage III Colon Cancer: A Nomogram Study. J Surg Res 2020;245:475-82.

11. Mirkin KA, Kulaylat AS, Hollenbeak CS, et al. Prognostic Significance of Tumor Deposits in Stage III Colon Cancer. Ann Surg Oncol 2018;25:3179-84.

12. Lo DS, Pollett A, Siu LL, et al. Prognostic significance of mesenteric tumor nodules in patients with stage III colorectal cancer. Cancer 2008;112:50-4.

13. Yabata E, Udagawa M, Okamoto H. Effect of tumor deposits on overall survival in colorectal cancer patients with regional lymph node metastases. J Rural Med 2014;9:20-6.

14. Puppa G, Maisonneuve P, Sonzogni A, et al. Pathological assessment of pericolonic tumor deposits in advanced colonic carcinoma: relevance to prognosis and tumor staging. Mod Pathol 2007;20:843-55.

15. Liu F, Zhao J, Li C, et al. The unique prognostic characteristics of tumor deposits in colorectal cancer patients. Ann Transl Med 2019;7:769.

16. Qin Q, Yang L, Zhou AP, et al. Prognostic value and initial exploratory research on TNM staging method of tumor deposits in stage III colon cancer. Zhonghua Wei Chang Wai Ke Za Zhi 2019;22:1152-8.

17. Rock JB, Washington MK, Adsay NV, et al. Debating deposits: an interobserver variability study of lymph nodes and pericolonic tumor deposits in colonic adenocarcinoma. Arch Pathol Lab Med 2014;138:636-42.

18. Song YX, Gao P, Wang ZN, et al. Can the tumor deposits be counted as metastatic lymph nodes in the UICC TNM staging system for colorectal cancer? PLoS One 2012;7:e34087.

19. Ueno H, Mochizuki H, Shirouzu K, et al. Multicenter study for optimal categorization of extramural tumor deposits for colorectal cancer staging. Ann Surg 2012;255:739-46.

20. Ueno H, Mochizuki H, Shirouzu K, et al. Actual status of distribution and prognostic impact of extramural discontinuous cancer spread in colorectal cancer. J Clin Oncol 2011;29:2550-6.

Cite this article as: Bai R, Tan Y, Li D, Yang M, Yu L, Yuan Y, Fang X. Development and validation of a novel prognostic nomogram including tumor deposits could better predict survival for colorectal cancer: a population-based study. Ann Transl Med 2021;9(8):620. doi: 10.21037/atm-20-4728 